\title{
General decay for Kirchhoff plates with a boundary condition of memory type
}

Jum-Ran Kang*

${ }^{*}$ Correspondence:

pointegg@hanmail.net

Department of Mathematics,

Dong-A University, Saha-Ku, Busan,

604-714, Korea

\begin{abstract}
In this paper we consider Kirchhoff plates with a memory condition at the boundary. For a wider class of relaxation functions, we establish a more general decay result, from which the usual exponential and polynomial decay rates are only special cases. MSC: 35B40; 74K20; 35L70
\end{abstract}

Keywords: Kirchhoff plates; general decay rate; memory term; relaxation function

\section{Introduction}

We consider the following Kirchhoff plates with a memory condition at the boundary:

$$
\begin{aligned}
& u_{t t}+\Delta^{2} u+a(x) u_{t}=0, \quad \text { in } \Omega \times(0, \infty), \\
& u=\frac{\partial u}{\partial v}=0, \quad \text { on } \Gamma_{0} \times(0, \infty), \\
& -u+\int_{0}^{t} g_{1}(t-s) \mathcal{B}_{2} u(s) d s=0, \quad \text { on } \Gamma_{1} \times(0, \infty), \\
& \frac{\partial u}{\partial v}+\int_{0}^{t} g_{2}(t-s) \mathcal{B}_{1} u(s) d s=0, \quad \text { on } \Gamma_{1} \times(0, \infty), \\
& u(0, x)=u_{0}(x), \quad u_{t}(0, x)=u_{1}(x), \quad \text { in } \Omega,
\end{aligned}
$$

where $a \in C^{1}(\bar{\Omega})$ and $\Omega$ is an open bounded set of $\mathbb{R}^{2}$ with a regular boundary $\Gamma$. We divide the boundary into two parts:

$$
\Gamma=\Gamma_{0} \cup \Gamma_{1} \quad \text { with } \bar{\Gamma}_{0} \cap \bar{\Gamma}_{1}=\emptyset \text {; and } \Gamma_{0} \neq \emptyset \text {. }
$$

Let us denote by $v=\left(v_{1}, v_{2}\right)$ the external unit normal to $\Gamma$, and let us denote by $\eta=\left(-v_{2}, v_{1}\right)$ the unit tangent positively oriented on $\Gamma$. We are denoting by $\mathcal{B}_{1}, \mathcal{B}_{2}$ the following differential operators:

$$
\mathcal{B}_{1} u=\Delta u+(1-\mu) B_{1} u, \quad \mathcal{B}_{2} u=\frac{\partial \Delta u}{\partial v}+(1-\mu) B_{2} u
$$

where $B_{1}$ and $B_{2}$ are given by

$$
B_{1} u=2 v_{1} v_{2} u_{x y}-v_{1}^{2} u_{y y}-v_{2}^{2} u_{x x}, \quad B_{2} u=\frac{\partial}{\partial \eta}\left[\left(v_{1}^{2}-v_{2}^{2}\right) u_{x y}+v_{1} v_{2}\left(u_{y y}-u_{x x}\right)\right]
$$

and the constant $\mu, 0<\mu<\frac{1}{2}$, represents Poisson's ratio.

\section{Springer}

(c) 2012 Kang; licensee Springer. This is an Open Access article distributed under the terms of the Creative Commons Attribution License (http://creativecommons.org/licenses/by/2.0), which permits unrestricted use, distribution, and reproduction in any medium, provided the original work is properly cited. 
In (1.1), $u$ denotes the position of the plate. The integral equations (1.3) and (1.4) describe the memory effects which can be caused, for example, by the interaction with another viscoelastic element. The relaxation functions $g_{1}, g_{2} \in C^{1}(0, \infty)$ are positive and nondecreasing. This system models the small transversal vibrations of a thin plate whose Poisson coefficient is equal to $\mu$. We assume that there exists $x_{0} \in R^{2}$ such that

$$
\begin{aligned}
& \Gamma_{0}=\left\{x \in \Gamma: v(x) \cdot\left(x-x_{0}\right) \leq 0\right\}, \\
& \Gamma_{1}=\left\{x \in \Gamma: v(x) \cdot\left(x-x_{0}\right)>0\right\} .
\end{aligned}
$$

If we denote the compactness of $\Gamma_{1}$ by $m(x)=x-x_{0}$, the condition (1.7) implies that there exists a small positive constant $\delta_{0}$ such that $0<\delta_{0} \leq m(x) \cdot v(x), \forall x \in \Gamma_{1}$.

The uniform stabilization of Kirchhoff plates with linear or nonlinear boundary feedback was investigated by several authors; see, for example, [1-3] among others. The uniform decay for plates with memory was studied in [4-6] and the references therein. There exists a large body of literature regarding viscoelastic problems with the memory term acting in the domain or at the boundary (see [7-12]). Rivera and Racke [13] investigated the decay results for magneto-thermo-elastic system. Santos et al. [14] studied the asymptotic behavior of the solutions of a nonlinear wave equation of Kirchhoff type with a boundary condition of memory type. Cavalcanti and Guesmia [15] proved the general decay rates of solutions to a nonlinear wave equation with a boundary condition of memory type. Park and Kang [16] studied the exponential decay for the multi-valued hyperbolic differential inclusion with a boundary condition of memory type. Kafini [17] showed the decay results for viscoelastic diffusion equations in the absence of instantaneous elasticity. They proved that the energy decays uniformly exponentially or algebraically at the same rate as the relaxation functions. In the present work, we generalize the earlier decay results of the solution of (1.1)-(1.5). More precisely, we show that the energy decays at the rate similar to the relaxation functions, which are not necessarily decaying like polynomial or exponential functions. In fact, our result allows a larger class of relaxation functions. Recently, Messaoudi and Soufyane [18], Santos and Soufyane [19], and Mustafa and Messaoudi [20] proved the general decay for the wave equation, von Karman plate system, and Timoshenko system with viscoelastic boundary conditions, respectively.

The paper is organized as follows. In Section 2 we present some notations and material needed for our work. In Section 3 we prove the general decay of the solutions to the Kirchhoff plates with a memory condition at the boundary.

\section{Preliminaries}

In this section, we present some material needed in the proof of our main result. We use the standard Lebesgue and Sobolev spaces with their usual scalar products and norms. Define the following space:

$$
W=\left\{w \in H^{2}(\Omega) ; w=\frac{\partial w}{\partial v}=0 \text { on } \Gamma_{0}\right\} .
$$

First, we shall use Eqs. (1.3) and (1.4) to estimate the values $\mathcal{B}_{1}$ and $\mathcal{B}_{2}$ on $\Gamma_{1}$. Denoting by

$$
(g * v)(t)=\int_{0}^{t} g(t-s) v(s) d s
$$


the convolution product operator and differentiating Eqs. (1.3) and (1.4), we arrive at the following Volterra equations:

$$
\begin{aligned}
& \mathcal{B}_{2} u+\frac{1}{g_{1}(0)} g_{1}^{\prime} * \mathcal{B}_{2} u=\frac{1}{g_{1}(0)} u_{t}, \\
& \mathcal{B}_{1} u+\frac{1}{g_{2}(0)} g_{2}^{\prime} * \mathcal{B}_{1} u=-\frac{1}{g_{2}(0)} \frac{\partial u_{t}}{\partial v} .
\end{aligned}
$$

Applying the Volterra inverse operator, we get

$$
\begin{aligned}
& \mathcal{B}_{2} u=\frac{1}{g_{1}(0)}\left\{u_{t}+k_{1} * u_{t}\right\}, \\
& \mathcal{B}_{1} u=-\frac{1}{g_{2}(0)}\left\{\frac{\partial u_{t}}{\partial v}+k_{2} * \frac{\partial u_{t}}{\partial v}\right\},
\end{aligned}
$$

where the resolvent kernels of $-\frac{g_{i}^{\prime}}{g_{i}(0)}$ satisfy

$$
k_{i}+\frac{1}{g_{i}(0)} g_{i}^{\prime} * k_{i}=-\frac{1}{g_{i}(0)} g_{i}^{\prime}, \quad \forall i=1,2 \text {. }
$$

Denoting by $\tau_{i}=\frac{1}{g_{i}(0)}$, for $i=1,2$, we obtain

$$
\begin{aligned}
& \mathcal{B}_{2} u=\tau_{1}\left\{u_{t}+k_{1}(0) u-k_{1}(t) u_{0}+k_{1}^{\prime} * u\right\}, \\
& \mathcal{B}_{1} u=-\tau_{2}\left\{\frac{\partial u_{t}}{\partial v}+k_{2}(0) \frac{\partial u}{\partial v}-k_{2}(t) \frac{\partial u_{0}}{\partial v}+k_{2}^{\prime} * \frac{\partial u}{\partial v}\right\} .
\end{aligned}
$$

Therefore, we use (2.1) and (2.2) instead of the boundary conditions (1.3) and (1.4).

Let us define the bilinear form $a(\cdot, \cdot)$ as follows:

$$
a(u, v)=\int_{\Omega}\left\{u_{x x} v_{x x}+u_{y y} v_{y y}+\mu\left(u_{x x} v_{y y}+u_{y y} v_{x x}\right)+2(1-\mu) u_{x y} v_{x y}\right\} d x d y .
$$

We state the following lemma which will be useful in what follows.

Lemma 2.1 ([2]) Let $u$ and $v$ be functions in $H^{4}(\Omega) \cap W$. Then we have

$$
\int_{\Omega}\left(\Delta^{2} u\right) v d x=a(u, v)+\int_{\Gamma_{1}}\left\{\left(\mathcal{B}_{2} u\right) v-\left(\mathcal{B}_{1} u\right) \frac{\partial v}{\partial v}\right\} d \Gamma .
$$

Let us denote

$$
(g \square v)(t):=\int_{0}^{t} g(t-s)|v(t)-v(s)|^{2} d s .
$$

The following lemma states an important property of the convolution operator.

Lemma 2.2 For $g, v \in C^{1}([0, \infty): \mathbb{R})$, we have

$$
(g * v) v_{t}=-\frac{1}{2} g(t)|v(t)|^{2}+\frac{1}{2} g^{\prime} \square v-\frac{1}{2} \frac{d}{d t}\left[g \square v-\left(\int_{0}^{t} g(s) d s\right)|v|^{2}\right] .
$$


The proof of this lemma follows by differentiating the term $g \square v$.

We formulate the following assumption:

(A1) Let $a \in C^{1}(\bar{\Omega})$ satisfy $a(x) \geq a_{0}>0$ in $\Omega$ for some $a_{0}$.

Let us introduce the energy function

$$
\begin{aligned}
E(t)= & \frac{1}{2}\left[\int_{\Omega}\left|u_{t}\right|^{2} d x+a(u, u)+\tau_{1} \int_{\Gamma_{1}}\left(k_{1}(t)|u|^{2}-k_{1}^{\prime} \square u\right) d \Gamma\right. \\
& \left.+\tau_{2} \int_{\Gamma_{1}}\left(k_{2}(t)\left|\frac{\partial u}{\partial v}\right|^{2}-k_{2}^{\prime} \square \frac{\partial u}{\partial v}\right) d \Gamma\right] .
\end{aligned}
$$

In these conditions, we are able to prove the existence of a strong solution.

Theorem 2.1 Let $k_{i} \in C^{2}\left(\mathbb{R}^{+}\right)$be such that

$$
\begin{gathered}
k_{i},-k_{i}^{\prime}, k_{i}^{\prime \prime} \geq 0 \quad(i=1,2) . \\
\text { If }\left(u_{0}, u_{1}\right) \in\left(H^{4}(\Omega) \cap W\right) \times W \text { satisfy the compatibility condition } \\
\mathcal{B}_{2} u_{0}-\tau_{1} u_{1}=0, \quad \mathcal{B}_{1} u_{0}+\tau_{2} \frac{\partial u_{1}}{\partial v} \quad \text { on } \Gamma_{1},
\end{gathered}
$$

then there is only one solution $u$ of the system (1.1)-(1.5) satisfying

$$
u \in L^{\infty}\left(0, T: H^{4}(\Omega) \cap W\right), \quad u_{t} \in L^{\infty}(0, T: W), \quad u_{t t} \in L^{\infty}\left(0, T: L^{2}(\Omega)\right) .
$$

Proof See Park and Kang [16].

\section{General decay}

In this section, we show that the solution of the system (1.1)-(1.5) may have a general decay not necessarily of exponential or polynomial type. For this we consider that the resolvent kernels satisfy the following hypothesis:

(H) $k_{i}: \mathbb{R}_{+} \rightarrow \mathbb{R}_{+}$is a twice differentiable function such that

$$
k_{i}(0)>0, \quad \lim _{t \rightarrow \infty} k_{i}(t)=0, \quad k_{i}^{\prime}(t) \leq 0,
$$

and there exists a nonincreasing continuous function $\xi_{i}: \mathbb{R}_{+} \rightarrow \mathbb{R}_{+}$satisfying

$$
k_{i}^{\prime \prime}(t) \geq-\xi_{i}(t) k_{i}^{\prime}(t), \quad i=1,2, \forall t \geq 0 .
$$

The following identity will be used later.

Lemma 3.1 ([2]) For every $v \in H^{4}(\Omega)$ and for every $\mu \in \mathbb{R}$, we have

$$
\begin{aligned}
\int_{\Omega}(m \cdot \nabla v) \Delta^{2} v d x= & a(v, v)+\frac{1}{2} \int_{\Gamma} m \cdot v\left[v_{x x}^{2}+v_{y y}^{2}+2 \mu v_{x x} v_{y y}+2(1-\mu) v_{x y}^{2}\right] d \Gamma \\
& +\int_{\Gamma}\left[\left(\mathcal{B}_{2} v\right) m \cdot \nabla v-\left(\mathcal{B}_{1} v\right) \frac{\partial}{\partial v}(m \cdot \nabla v)\right] d \Gamma .
\end{aligned}
$$


Our point of departure will be to establish some inequalities for the strong solution of the system (1.1)-(1.5).

Lemma 3.2 The energy functional E satisfies, along the solution of (1.1)-(1.5), the estimate

$$
\begin{aligned}
E^{\prime}(t) \leq & -\int_{\Omega} a(x)\left|u_{t}\right|^{2} d x-\frac{\tau_{1}}{2} \int_{\Gamma_{1}}\left(\left|u_{t}\right|^{2}-k_{1}^{2}(t)\left|u_{0}\right|^{2}-k_{1}^{\prime}(t)|u|^{2}+k_{1}^{\prime \prime} \square u\right) d \Gamma \\
& -\frac{\tau_{2}}{2} \int_{\Gamma_{1}}\left(\left|\frac{\partial u_{t}}{\partial v}\right|^{2}-k_{2}^{2}(t)\left|\frac{\partial u_{0}}{\partial v}\right|^{2}-k_{2}^{\prime}(t)\left|\frac{\partial u}{\partial v}\right|^{2}+k_{2}^{\prime \prime} \square \frac{\partial u}{\partial v}\right) d \Gamma .
\end{aligned}
$$

Proof Multiplying Eq. (1.1) by $u_{t}$, integrating over $\Omega$, and using Lemma 2.1, we get

$$
\frac{1}{2} \frac{d}{d t}\left\{\int_{\Omega}\left|u_{t}\right|^{2} d x+a(u, u)\right\}=-\int_{\Gamma_{1}}\left(\mathcal{B}_{2} u\right) u_{t} d \Gamma+\int_{\Gamma_{1}}\left(\mathcal{B}_{1} u\right) \frac{\partial u_{t}}{\partial v} d \Gamma-\int_{\Omega} a(x)\left|u_{t}\right|^{2} d x
$$

Substituting the boundary terms by (2.1) and (2.2) and using Lemma 2.2 and the Young inequality, our conclusion follows.

Let us consider the following binary operator:

$$
(k \circ \varphi)(t):=\int_{0}^{t} k(t-s)(\varphi(t)-\varphi(s)) d s
$$

Then applying the Holder inequality for $0 \leq \alpha \leq 1$, we have

$$
|(k \circ \varphi)(t)|^{2} \leq\left[\int_{0}^{t}|k(s)|^{2(1-\alpha)} d s\right]\left(|k|^{2 \alpha} \square \varphi\right)(t) .
$$

Let us define the functional

$$
\psi(t)=\int_{\Omega}(m \cdot \nabla u) u_{t} d x
$$

The following lemma plays an important role in the construction of the desired functional.

Lemma 3.3 Suppose that the initial data $\left(u_{0}, u_{1}\right) \in\left(H^{4}(\Omega) \cap W\right) \times W$ and satisfies the compatibility condition (2.5). Then the solution of the system (1.1)-(1.5) satisfies

$$
\begin{aligned}
\frac{d}{d t} \psi(t) \leq & \frac{1}{2} \int_{\Gamma_{1}} m \cdot v\left|u_{t}\right|^{2} d \Gamma-\frac{1}{2} \int_{\Omega}\left|u_{t}\right|^{2} d x \\
& -\left(1-\frac{C_{0}}{2}-\frac{\epsilon \lambda_{0}}{2}\right) a(u, u)+\frac{1}{2} \int_{\Omega} a(x)\left|u_{t}\right|^{2} d x \\
& +\frac{2 \tau_{1}^{2}}{\epsilon} \int_{\Gamma_{1}}\left\{\left|u_{t}\right|^{2}+k_{1}^{2}(t)|u|^{2}+k_{1}^{2}(t)\left|u_{0}\right|^{2}+\left|k_{1}^{\prime} \circ u\right|^{2}\right\} d \Gamma \\
& +\frac{2 \tau_{2}^{2}}{\epsilon} \int_{\Gamma_{1}}\left\{\left|\frac{\partial u_{t}}{\partial v}\right|^{2}+k_{2}^{2}(t)\left|\frac{\partial u}{\partial v}\right|^{2}+k_{2}^{2}(t)\left|\frac{\partial u_{0}}{\partial v}\right|^{2}+\left|k_{2}^{\prime} \circ \frac{\partial u}{\partial v}\right|^{2}\right\} d \Gamma \\
& -\left(\frac{1}{2}-\frac{\epsilon \lambda_{0}}{2 \delta_{0}}\right) \int_{\Gamma_{1}} m \cdot v\left[u_{x x}^{2}+u_{y y}^{2}+2 \mu u_{x x} u_{y y}+2(1-\mu) u_{x y}^{2}\right] d \Gamma .
\end{aligned}
$$


Proof Differentiating $\psi$, using Eq. (1.1), and taking $v=u$ in Lemma 3.1, we get

$$
\begin{aligned}
\frac{d}{d t} \psi(t)= & \int_{\Omega}\left(m \cdot \nabla u_{t}\right) u_{t} d x+\int_{\Omega}(m \cdot \nabla u) u_{t t} d x \\
= & \frac{1}{2} \int_{\Gamma_{1}} m \cdot v\left|u_{t}\right|^{2} d \Gamma-\int_{\Omega}\left|u_{t}\right|^{2} d x-a(u, u) \\
& -\int_{\Gamma}\left[\left(\mathcal{B}_{2} u\right)(m \cdot \nabla u)-\left(\mathcal{B}_{1} u\right) \frac{\partial}{\partial v}(m \cdot \nabla u)\right] d \Gamma \\
& -\frac{1}{2} \int_{\Gamma} m \cdot v\left[u_{x x}^{2}+u_{y y}^{2}+2 \mu u_{x x} u_{y y}+2(1-\mu) u_{x y}^{2}\right] d \Gamma \\
& -\int_{\Omega} a(x) u_{t}(m \cdot \nabla u) d x .
\end{aligned}
$$

Let us next examine the integrals over $\Gamma_{0}$ in (3.4). Since $u=\frac{\partial u}{\partial v}=0$ on $\Gamma_{0}$, we have $B_{1} u=$ $B_{2} u=0$ on $\Gamma_{0}$ and

$$
\begin{aligned}
& \frac{\partial}{\partial v}(m \cdot \nabla u)=(m \cdot v) \Delta u, \\
& u_{x x}^{2}+u_{y y}^{2}+2 \mu u_{x x} u_{y y}+2(1-\mu) u_{x y}^{2}=(\Delta u)^{2} \quad \text { on } \Gamma_{0}
\end{aligned}
$$

since

$$
u_{x x} u_{y y}-\left(u_{x y}\right)^{2}=0 \quad \text { on } \Gamma_{0} .
$$

Therefore, from (3.4) and (3.5), we have

$$
\begin{aligned}
\frac{d}{d t} \psi(t) \leq & \frac{1}{2} \int_{\Gamma_{1}} m \cdot v\left|u_{t}\right|^{2} d \Gamma-\int_{\Omega}\left|u_{t}\right|^{2} d x-a(u, u)+\frac{1}{2} \int_{\Gamma_{0}} m \cdot v(\Delta u)^{2} d \Gamma \\
& -\frac{1}{2} \int_{\Gamma_{1}} m \cdot v\left[u_{x x}^{2}+u_{y y}^{2}+2 \mu u_{x x} u_{y y}+2(1-\mu) u_{x y}^{2}\right] d \Gamma \\
& -\int_{\Gamma_{1}}\left(\mathcal{B}_{2} u\right)(m \cdot \nabla u) d \Gamma+\int_{\Gamma_{1}}\left(\mathcal{B}_{1} u\right) \frac{\partial}{\partial v}(m \cdot \nabla u) d \Gamma \\
& -\int_{\Omega} a(x) u_{t}(m \cdot \nabla u) d x .
\end{aligned}
$$

Using the Young inequality, we have

$$
\begin{aligned}
& \left|\int_{\Gamma_{1}}\left(\mathcal{B}_{2} u\right)(m \cdot \nabla u) d \Gamma\right| \leq \frac{1}{2 \epsilon} \int_{\Gamma_{1}}\left|\mathcal{B}_{2} u\right|^{2} d \Gamma+\frac{\epsilon}{2} \int_{\Gamma_{1}}|m \cdot \nabla u|^{2} d \Gamma \\
& \left|\int_{\Gamma_{1}}\left(\mathcal{B}_{1} u\right) \frac{\partial}{\partial \nu}(m \cdot \nabla u) d \Gamma\right| \leq \frac{1}{2 \epsilon} \int_{\Gamma_{1}}\left|\mathcal{B}_{1} u\right|^{2} d \Gamma+\frac{\epsilon}{2} \int_{\Gamma_{1}}\left|\frac{\partial}{\partial \nu}(m \cdot \nabla u)\right|^{2} d \Gamma,
\end{aligned}
$$

where $\epsilon$ is a positive constant. Since the bilinear form $a(u, u)$ is strictly coercive on $W$, using the trace theory, we obtain

$$
\begin{aligned}
& \int_{\Gamma_{1}}|m \cdot \nabla u|^{2} d \Gamma+\int_{\Gamma_{1}}\left|\frac{\partial}{\partial v}(m \cdot \nabla u)\right|^{2} d \Gamma \\
& \quad \leq \lambda_{0} a(u, u)+\frac{\lambda_{0}}{\delta_{0}} \int_{\Gamma_{1}} m \cdot v\left[u_{x x}^{2}+u_{y y}^{2}+2 \mu u_{x x} u_{y y}+2(1-\mu) u_{x y}^{2}\right] d \Gamma
\end{aligned}
$$


where $\lambda_{0}$ is a constant depending on $\Omega$ and $\mu$. Further, one has

$$
\left|\int_{\Omega} a(x) u_{t}(m \cdot \nabla u) d x\right| \leq \frac{1}{2} \int_{\Omega} a(x)\left|u_{t}\right|^{2} d x+\frac{C_{0}}{2} a(u, u)
$$

where $\int_{\Omega}|\nabla u|^{2} d x \leq C_{0} a(u, u)$ with some constant $C_{0}>0$. Substituting the inequalities (3.7)-(3.10) into (3.6) and taking into account the fact that $m \cdot v \leq 0$ on $\Gamma_{0}$, we have

$$
\begin{aligned}
\frac{d}{d t} \psi(t) \leq & \frac{1}{2} \int_{\Gamma_{1}} m \cdot v\left|u_{t}\right|^{2} d \Gamma-\frac{1}{2} \int_{\Omega}\left|u_{t}\right|^{2} d x \\
& -\left(1-\frac{C_{0}}{2}-\frac{\epsilon \lambda_{0}}{2}\right) a(u, u)+\frac{1}{2 \epsilon} \int_{\Gamma_{1}}\left|\mathcal{B}_{1} u\right|^{2}+\left|\mathcal{B}_{2} u\right|^{2} d \Gamma \\
& -\left(\frac{1}{2}-\frac{\epsilon \lambda_{0}}{2 \delta_{0}}\right) \int_{\Gamma_{1}} m \cdot v\left[u_{x x}^{2}+u_{y y}^{2}+2 \mu u_{x x} u_{y y}+2(1-\mu) u_{x y}^{2}\right] d \Gamma \\
& +\frac{1}{2} \int_{\Omega} a(x)\left|u_{t}\right|^{2} d x .
\end{aligned}
$$

Since the boundary conditions (2.1) and (2.2) can be written as

$$
\begin{aligned}
& \mathcal{B}_{2} u=\tau_{1}\left\{u_{t}+k_{1}(t) u-k_{1}(t) u_{0}-k_{1}^{\prime} \circ u\right\}, \\
& \mathcal{B}_{1} u=-\tau_{2}\left\{\frac{\partial u_{t}}{\partial v}+k_{2}(t) \frac{\partial u}{\partial v}-k_{2}(t) \frac{\partial u_{0}}{\partial v}-k_{2}^{\prime} \circ \frac{\partial u}{\partial v}\right\},
\end{aligned}
$$

our conclusion follows.

Let us introduce the Lyapunov functional

$$
\mathcal{L}(t)=N E(t)+\psi(t)
$$

with $N>0$. Now we are in a position to show the main result of this paper.

Theorem 3.1 Let $\left(u_{0}, u_{1}\right) \in W \times L^{2}(\Omega)$. Suppose the resolvent kernels $k_{1}, k_{2}$ satisfy the conditions $(\mathrm{H})$ and (3.1). Then there exist constants $\omega, C>0$ such that for some $t_{0}$ large enough, the solution of (1.1)-(1.5) satisfies

$$
E(t) \leq C E(0) e^{-\omega \int_{0}^{t} \xi(s) d s}, \quad \forall t \geq t_{0}, \text { if } u_{0}=\frac{\partial u_{0}}{\partial v}=0 \text { on } \Gamma_{1} .
$$

Otherwise,

$$
E(t) \leq C\left(E(0)+\int_{0}^{t} k_{0}(s) e^{\omega \int_{t_{0}}^{s} \xi(\tau) d \tau} d s\right) e^{-\omega \int_{0}^{t} \xi(s) d s}
$$

for all $t \geq t_{0}$, where

$$
\xi(t)=\min \left\{\xi_{1}(t), \xi_{2}(t)\right\}
$$

and

$$
k_{0}(t)=\int_{\Gamma_{1}} k_{1}^{2}(t)\left|u_{0}\right|^{2} d \Gamma+\int_{\Gamma_{1}} k_{2}^{2}(t)\left|\frac{\partial u_{0}}{\partial v}\right|^{2} d \Gamma .
$$


Proof Applying the inequality (3.3) with $\alpha=\frac{1}{2}$ in Lemma 3.3 and from Lemma 3.2, we obtain

$$
\begin{aligned}
\mathcal{L}^{\prime}(t) \leq & -\left(N-\frac{1}{2}\right) \int_{\Omega} a(x)\left|u_{t}\right|^{2} d x \\
& -\frac{\tau_{1} N}{2} \int_{\Gamma_{1}}\left\{\left|u_{t}\right|^{2}-k_{1}^{2}(t)\left|u_{0}\right|^{2}-k_{1}^{\prime}(t)|u|^{2}+k_{1}^{\prime \prime} \square u\right\} d \Gamma \\
& -\frac{\tau_{2} N}{2} \int_{\Gamma_{1}}\left\{\left|\frac{\partial u_{t}}{\partial v}\right|^{2}-k_{2}^{2}(t)\left|\frac{\partial u_{0}}{\partial v}\right|^{2}-k_{2}^{\prime}(t)\left|\frac{\partial u}{\partial v}\right|^{2}+k_{2}^{\prime \prime} \square \frac{\partial u}{\partial v}\right\} d \Gamma \\
& -\left(1-\frac{C_{0}}{2}-\frac{\epsilon \lambda_{0}}{2}\right) a(u, u)+\frac{1}{2} \int_{\Gamma_{1}} m \cdot v\left|u_{t}\right|^{2} d \Gamma \\
& +\frac{2 \tau_{1}^{2}}{\epsilon} \int_{\Gamma_{1}}\left\{\left|u_{t}\right|^{2}+k_{1}^{2}(t)|u|^{2}+k_{1}^{2}(t)\left|u_{0}\right|^{2}-k_{1}(0) k_{1}^{\prime} \square u\right\} d \Gamma \\
& +\frac{2 \tau_{2}^{2}}{\epsilon} \int_{\Gamma_{1}}\left\{\left|\frac{\partial u_{t}}{\partial v}\right|^{2}+k_{2}^{2}(t)\left|\frac{\partial u}{\partial v}\right|^{2}+k_{2}^{2}(t)\left|\frac{\partial u_{0}}{\partial v}\right|^{2}-k_{2}(0) k_{2}^{\prime} \square \frac{\partial u}{\partial v}\right\} d \Gamma \\
& -\frac{1}{2} \int_{\Omega}\left|u_{t}\right|^{2} d x-\left(\frac{1}{2}-\frac{\epsilon \lambda_{0}}{2 \delta_{0}}\right) \\
& \times \int_{\Gamma_{1}} m \cdot v\left[u_{x x}^{2}+u_{y y}^{2}+2 \mu u_{x x} u_{y y}+2(1-\mu) u_{x y}^{2}\right] d \Gamma .
\end{aligned}
$$

Then, choosing $N$ large enough and $0<\epsilon<\min \left\{\frac{2-C_{0}}{\lambda_{0}}, \frac{\delta_{0}}{\lambda_{0}}\right\}$, we obtain

$$
\begin{aligned}
\mathcal{L}^{\prime}(t) \leq & -c_{0} E(t)+c \int_{\Gamma_{1}} k_{1}^{2}(t)\left|u_{0}\right|^{2} d \Gamma+c \int_{\Gamma_{1}} k_{2}^{2}(t)\left|\frac{\partial u_{0}}{\partial v}\right|^{2} d \Gamma \\
& -c \int_{\Gamma_{1}} k_{1}^{\prime} \square u d \Gamma-c \int_{\Gamma_{1}} k_{2}^{\prime} \square \frac{\partial u}{\partial v} d \Gamma, \quad \forall t \geq t_{0} .
\end{aligned}
$$

On the other hand, we can choose $N$ even larger so that

$$
\mathcal{L}(t) \sim E(t)
$$

If $\xi(t)=\min \left\{\xi_{1}(t), \xi_{2}(t)\right\}, t \geq 0$, then using (3.1) and (3.2), we have

$$
\begin{aligned}
\xi(t) \mathcal{L}^{\prime}(t) \leq & -c_{0} \xi(t) E(t)+c \xi(t) \int_{\Gamma_{1}} k_{1}^{2}(t)\left|u_{0}\right|^{2} d \Gamma+c \xi(t) \int_{\Gamma_{1}} k_{2}^{2}(t)\left|\frac{\partial u_{0}}{\partial v}\right|^{2} d \Gamma \\
& -c \xi(t) \int_{\Gamma_{1}} k_{1}^{\prime} \square u d \Gamma-c \xi_{2}(t) \int_{\Gamma_{1}} k_{2}^{\prime} \square \frac{\partial u}{\partial v} d \Gamma \\
\leq & -c_{0} \xi(t) E(t)+c \xi(t) \int_{\Gamma_{1}} k_{1}^{2}(t)\left|u_{0}\right|^{2} d \Gamma+c \xi(t) \int_{\Gamma_{1}} k_{2}^{2}(t)\left|\frac{\partial u_{0}}{\partial v}\right|^{2} d \Gamma \\
& +c \int_{\Gamma_{1}} k_{1}^{\prime \prime} \square u d \Gamma+c \int_{\Gamma_{1}} k_{2}^{\prime \prime} \square \frac{\partial u}{\partial v} d \Gamma \\
\leq & -c_{0} \xi(t) E(t)+c \xi(t) \int_{\Gamma_{1}} k_{1}^{2}(t)\left|u_{0}\right|^{2} d \Gamma \\
& +c \xi(t) \int_{\Gamma_{1}} k_{2}^{2}(t)\left|\frac{\partial u_{0}}{\partial v}\right|^{2} d \Gamma-c E^{\prime}(t), \quad \forall t \geq t_{0},
\end{aligned}
$$


which gives

$$
\begin{aligned}
& \xi(t) \mathcal{L}^{\prime}(t)+c E^{\prime}(t) \\
& \quad \leq-c_{0} \xi(t) E(t)+c \xi(t) \int_{\Gamma_{1}} k_{1}^{2}(t)\left|u_{0}\right|^{2} d \Gamma+c \xi(t) \int_{\Gamma_{1}} k_{2}^{2}(t)\left|\frac{\partial u_{0}}{\partial v}\right|^{2} d \Gamma, \quad \forall t \geq t_{0} .
\end{aligned}
$$

Using the fact that $\xi$ is a nonincreasing continuous function as $\xi_{1}$ and $\xi_{2}$ are nonincreasing, and so $\xi$ is differentiable, with $\xi^{\prime}(t) \leq 0$, for a.e. $t$, then we infer that

$$
\begin{aligned}
(\xi \mathcal{L} & +c E)^{\prime}(t) \\
& \leq \xi(t) \mathcal{L}^{\prime}(t)+c E^{\prime}(t) \\
& \leq-c_{0} \xi(t) E(t)+c \xi(t) \int_{\Gamma_{1}} k_{1}^{2}(t)\left|u_{0}\right|^{2} d \Gamma+c \xi(t) \int_{\Gamma_{1}} k_{2}^{2}(t)\left|\frac{\partial u_{0}}{\partial v}\right|^{2} d \Gamma, \quad \forall t \geq t_{0} .
\end{aligned}
$$

Since using (3.14),

$$
F=\xi \mathcal{L}+c E \sim E
$$

we obtain for some positive constant $\omega$,

$$
F^{\prime}(t) \leq-\omega \xi(t) F(t)+c \int_{\Gamma_{1}} k_{1}^{2}(t)\left|u_{0}\right|^{2} d \Gamma+c \int_{\Gamma_{1}} k_{2}^{2}(t)\left|\frac{\partial u_{0}}{\partial v}\right|^{2} d \Gamma, \quad \forall t \geq t_{0} .
$$

Case 1: If $u_{0}=\frac{\partial u_{0}}{\partial v}=0$ on $\Gamma_{1}$, then (3.16) reduces to

$$
F^{\prime}(t) \leq-\omega \xi(t) F(t), \quad \forall t \geq t_{0} .
$$

A simple integration over $\left(t_{0}, t\right)$ yields

$$
F(t) \leq F\left(t_{0}\right) e^{-\omega \int_{t_{0}}^{t} \xi(s) d s}, \quad \forall t \geq t_{0} .
$$

By using (3.2) and (3.15), we then obtain for some positive constant $C$

$$
E(t) \leq C E(0) e^{-\omega \int_{0}^{t} \xi(s) d s}, \quad \forall t \geq t_{0} .
$$

Thus, the estimate (3.11) is proved.

Case 2: If $\left(u_{0}, \frac{\partial u_{0}}{\partial v}\right) \neq(0,0)$ on $\Gamma_{1}$, then (3.16) gives

$$
F^{\prime}(t) \leq-\omega \xi(t) F(t)+c k_{0}(t), \quad \forall t \geq t_{0}
$$

where

$$
k_{0}(t)=\int_{\Gamma_{1}} k_{1}^{2}(t)\left|u_{0}\right|^{2} d \Gamma+\int_{\Gamma_{1}} k_{2}^{2}(t)\left|\frac{\partial u_{0}}{\partial v}\right|^{2} d \Gamma .
$$

In this case, we introduce

$$
G(t):=F(t)-c e^{-\omega \int_{t_{0}}^{t} \xi(s) d s} \int_{t_{0}}^{t} k_{0}(s) e^{\omega \int_{t_{0}}^{s} \xi(\tau) d \tau} d s .
$$


A simple differentiation of $G$, using (3.17), leads to

$$
G^{\prime}(t)=F^{\prime}(t)+\omega \xi(t) c e^{-\omega \int_{t_{0}}^{t} \xi(s) d s} \int_{t_{0}}^{t} k_{0}(s) e^{\omega \int_{t_{0}}^{s} \xi(\tau) d \tau} d s-c k_{0}(t) \leq-\omega \xi(t) G(t), \quad \forall t \geq t_{0}
$$

Again, a simple integration over $\left(t_{0}, t\right)$ yields

$$
G(t) \leq G\left(t_{0}\right) e^{-\omega \int_{t_{0}}^{t} \xi(s) d s}, \quad \forall t \geq t_{0}
$$

which implies, for all $t \geq t_{0}$,

$$
F(t) \leq\left(F\left(t_{0}\right)+c \int_{t_{0}}^{t} k_{0}(s) e^{\omega \int_{t_{0}}^{s} \xi(\tau) d \tau} d s\right) e^{-\omega \int_{t_{0}}^{t} \xi(s) d s}
$$

By using (3.15), we deduce

$$
E(t) \leq C\left(E(0)+\int_{0}^{t} k_{0}(s) e^{\omega \int_{t_{0}}^{s} \xi(\tau) d \tau} d s\right) e^{-\omega \int_{t_{0}}^{t} \xi(s) d s}, \quad \forall t \geq t_{0}
$$

Consequently, by the boundedness of $\xi$, (3.12) is established.

Remark 3.1 Estimates (3.11) and (3.12) are also true for $t \in\left[0, t_{0}\right]$ by virtue of continuity and boundedness of $E(t)$ and $\xi$.

Remark 3.2 Note that the exponential and polynomial decay estimates are only particular cases of (3.11) and (3.12). More precisely, we have exponential decay for $\xi_{1}(t) \equiv c_{1}$ and $\xi_{2}(t) \equiv c_{2}$ and polynomial decay for $\xi_{1}(t)=c_{1}(1+t)^{-1}$ and $\xi_{2}(t) \equiv c_{2}$, where $c_{1}$ and $c_{2}$ are positive constants.

Example 3.1 As in [20], we give some examples to illustrate the energy decay rates given by $(3.11)$.

(1) If $k_{1}(t)=k_{2}(t)=a e^{-b(1+t)^{p}}, 0<p \leq 1$, then for $i=1,2, k_{i}^{\prime \prime}(t) \geq-\xi(t) k_{i}^{\prime}(t)$, where $\xi(t)=$ $b p(1+t)^{p-1}$. For suitably chosen positive constants $a$ and $b, k_{i}$ satisfies $(\mathrm{H})$ and (3.11) gives

$$
E(t) \leq c e^{-\omega b(1+t)^{p}}
$$

(2) If $k_{1}(t)=\frac{a_{1}}{(1+t)^{q}}, q>0$, and $k_{2}(t)=a_{2} e^{-b(1+t)^{p}}, 0<p \leq 1$, then for $i=1,2, k_{i}^{\prime \prime}(t) \geq$ $-\xi(t) k_{i}^{\prime}(t)$, where $\xi(t)=q(1+t)^{-1}$. Then

$$
E(t) \leq \frac{c}{(1+t)^{\omega q}}
$$

The above two examples are included in the following more general one.

(3) For any nonincreasing functions $k_{i}(t), i=1,2$, which satisfy $(\mathrm{H}), \xi_{i}=-\frac{k^{\prime}}{k}$ are also nonincreasing differentiable functions, and $c \xi_{1}(t) \leq \xi_{2}(t)$, for some $0<c \leq 1$, (3.11) gives

$$
E(t) \leq c\left[k_{1}(t)\right]^{\omega}
$$


Author's contributions

The work was realized by the author.

\section{Acknowledgements}

The author thanks the anonymous referee for a careful review. This work was supported by the Dong-A University research fund.

Received: 30 July 2012 Accepted: 25 October 2012 Published: 7 November 2012

\section{References}

1. Komornik, V: On the nonlinear boundary stabilization of Kirchhoff plates. Nonlinear Differ. Equ. Appl. 1, 323-337 (1994)

2. Lagnese, JE: Boundary Stabilization of Thin Plates. SIAM, Philadelphia (1989)

3. Lasiecka, I: Exponential decay rates for the solutions of Euler-Bernoulli moments only. J. Differ. Equ. 95, 169-182 (1992)

4. Lagnese, JE: Asymptotic Energy Estimates for Kirchhoff Plates Subject to Weak Viscoelastic Damping. International Series of Numerical Mathematics, vol. 91. Birkhäuser, Bassel (1989)

5. Munoz Rivera, JE, Lapa, EC, Barreto, R: Decay rates for viscoelastic plates with memory. J. Elast. 44, 61-87 (1996)

6. Santos, ML, Junior, F: A boundary condition with memory for Kirchhoff plates equations. Appl. Math. Comput. 148, 475-496 (2004)

7. Aassila, M, Cavalcanti, MM, Soriano, JA: Asymptotic stability and energy decay rates for solutions of the wave equation with memory in a star-shaped domain. SIAM J. Control Optim. 38(5), 1581-1602 (2000)

8. Cavalcanti, MM, Domingos Cavalcanti, VN, Soriano, JA: Exponential decay for the solution of semilinear viscoelastic wave equation with localized damping. Electron. J. Differ. Equ. 2002, 44 (2002)

9. Cavalcanti, MM, Domingos Cavalcanti, VN, Martinez, P: General decay rate estimates for viscoelastic dissipative systems. Nonlinear Anal. 68(1), 177-193 (2008)

10. Kang, JR: Energy decay rates for von Kármán system with memory and boundary feedback. Appl. Math. Comput. 218, 9085-9094 (2012)

11. Messaoudi, SA: General decay of solutions of a viscoelastic equation. J. Math. Anal. Appl. 341, 1457-1467 (2008)

12. Messaoudi, SA, Tatar, NE: Exponential and polynomial decay for a quasilinear viscoelastic equation. Nonlinear Anal. 68, 785-793 (2008)

13. Munoz Rivera, JE, Racke, R: Magneto-thermo-elasticity: large time behavior for linear systems. Adv. Differ. Equ. 6(3), 359-384 (2001)

14. Santos, ML, Ferreira, J, Pereira, DC, Raposo, CA: Global existence and stability for wave equation of Kirchhoff type with memory condition at the boundary. Nonlinear Anal. 54, 959-976 (2003)

15. Cavalcanti, MM, Guesmia, A: General decay rates of solutions to a nonlinear wave equation with boundary condition of memory type. Differ. Integral Equ. 18(5), 583-600 (2005)

16. Park, JY, Kang, JR: A boundary condition with memory for the Kirchhoff plate equations with non-linear dissipation. Math. Methods Appl. Sci. 29, 267-280 (2006)

17. Kafini, M: Decay results for viscoelastic diffusion equations in absence of instantaneous elasticity. Electron. J. Differ Equ. 2012, 73 (2012)

18. Messaoudi, SA, Soufyane, A: General decay of solutions of a wave equation with a boundary control of memory type. Nonlinear Anal., Real World Appl. 11, 2896-2904 (2010)

19. Santos, ML, Soufyane, A: General decay to a von Kármán plate system with memory boundary conditions. Differ. Integral Equ. 24(1-2), 69-81 (2011)

20. Mustafa, MI, Messaoudi, SA: Energy decay rates for a Timoshenko system with viscoelastic boundary conditions. Appl. Math. Comput. 218, 9125-9131 (2012)

doi:10.1186/1687-2770-2012-129

Cite this article as: Kang: General decay for Kirchhoff plates with a boundary condition of memory type. Boundary Value Problems 2012 2012:129. 